\begin{tabular}{|c|c|c|c|c|}
\hline \multicolumn{5}{|c|}{ Table comparing Initial audit and subsequent PDSA cycles } \\
\hline & $\begin{array}{l}\text { No. of } \\
\text { patients }\end{array}$ & $\begin{array}{l}\text { \% of those } \\
\text { with } \mathrm{AF}\end{array}$ & $\begin{array}{l}\% \text { of those with } \\
\mathrm{AF} \text { on } \\
\text { anticoagulation }\end{array}$ & $\begin{array}{c}\text { AF box } \\
\text { completed }\end{array}$ \\
\hline Initial audit & 166 & $36 \%$ & $33 \%$ & $\mathrm{n} / \mathrm{a}$ \\
\hline $\begin{array}{l}\text { 1st PDSA } \\
\text { Cycle March }\end{array}$ & 38 & $40 \%$ & $60 \%$ & $39 \%$ \\
\hline $\begin{array}{l}\text { 2nd PDSA } \\
\text { cycle April }\end{array}$ & 47 & $28 \%$ & $23 \%$ & $51 \%$ \\
\hline $\begin{array}{l}\text { 3rd PDSA } \\
\text { cycle May }\end{array}$ & 38 & $32 \%$ & $50 \%$ & $71 \%$ \\
\hline
\end{tabular}

and exclusion criteria, wide dissemination of these criteria across the cardiac/stroke network, and instituting a dedicated LAAO clinic for patient assessment pre- and post-implant.

Results Between August 2014 and November 2015, 74 LAAO referrals were reviewed at MDT. LAAO was offered to 52/74 $(70.3 \%)$ patients, of which $42(56.8 \%)$ underwent the procedure, $8(10.8 \%)$ are awaiting it, while $2(2.7 \%)$ declined consent. A decision was taken to treat $14(18.9 \%)$ patients with a novel oral anticoagulant (NOAC) and to maintain watchful waiting in $5(6.8 \%)$. $3(4.1 \%)$ referrals were felt to be inappropriate.

42 patients (mean age $75 \pm 9$ years, 25 males, median $\mathrm{CHA}_{2} \mathrm{DS}_{2}$ VASc score 4 , median HAS-BLED score 2) underwent LAAO under GA and TOE guidance. The Amplatzer Cardiac Plug was used in the initial 3 cases and Amplatzer Amulet in the remaining 39. Implant was successful in all cases. Mean procedure time was $93 \pm 229$ min and mean flouro time was $14 \pm 88 \mathrm{~min}$. Periprocedural haemorrhage requiring blood transfusion occurred in 2 (4.8\%) cases: no other complications were observed. Patients were discharged on a 6-week course of dual antiplatelet therapy, followed by therapy with a single antiplatelet agent.

To date, clinical follow-up is available for 27 patients, while most recent 14 patients are awaiting their first follow up; 1 patient has been lost to follow-up. At mean follow-up of $189 \pm 119$ days, $25 / 27$ patients $(92.6 \%)$ are free of haemorrhage or thromboembolic events, 1 patient suffered stroke at 12 months post-LAAO, and 1 patient died 3 weeks after the procedure. 24 patients to date have undergone follow-up imaging, 16 patients are awaiting it, and 1 patient could not tolerate TOE and is awaiting CT. 22 of these $24(91.6 \%)$ patients imaged had good LAA seal with no residual leak, while $2(8 \%)$ had small $(<5 \mathrm{~mm})$ leak. 1 patient had evidence of a laminar clot on the device and was started on a NOAC. Conclusions Contemporary LAAO implant is associated with $100 \%$ procedural success, a very low rate of complications and a high rate of LAA seal on follow up imaging. At our centre, the $\mathrm{CtE}$ process has been a catalyst for change and has led to streamlining processes along the entire patient journey. With good clinician engagement, it should provide informative real-life data on patient outcomes that could be used to make funding decisions for the wider NHS.

\section{IMPROVING SAFETY AND CLINICAL OUTCOMES FOR ATRIAL FIBRILLATION ABLATION: SHOULD OUR THRESHOLD FOR REFERRAL AND FOR INTERVENTION BECOME LOWER?}

${ }^{1}$ Yawer Saeed ${ }^{*},{ }^{1}$ Ahmed Hussein, ${ }^{1}$ Wern Ding, 'Lisa McClenaghan, ${ }^{1}$ Mohamed Meah ${ }^{2}$ Richard Snowdon, ${ }^{2}$ Mark Hall, ${ }^{2}$ Johan Waktare, ${ }^{2}$ Simon Modi, ${ }^{2}$ Derick Todd, ${ }^{1}$ Dhiraj Gupta. ${ }^{1}$ Liverpool Heart and Chest Hospital, NHS Foundation; ${ }^{2}$ Liverpool Heart and Chest Hospital; *Presenting Author

\subsection{6/heartjnl-2016-309890.63}

Introduction Historically, the significant risk of complications associated with AF ablation (AFA) has been a potential deterrent for referrers and patients alike. Ours is a high volume EP centre with a fully integrated Electronic Patient Record System that allows for comprehensive data capture and retrieval. We sought to assess success and complication rates of contemporary AFA practice.

Methods We assessed safety and clinical outcome data on follow up for consecutive patients who underwent AFA between Sep 2011 and Aug 2013. A total of 659 AFAs were performed over this period by 6 operators, with 95\% done using Carto mapping and a contact force sensing RF catheter. Vascular ultrasound to guide femoral vein access was used in all patients, and general anaesthesia was used in $25 \%$. All patients on prior Warfarin had this continued peri-procedure, with no bridging Heparin used in any case.

Clinical follow up data were evaluated for the 425 consecutive patients $(69 \%$ male, $59.9 \pm 10.4$ years) who were first time AFAs over this period. Of these, 283 (66.8\%) had paroxysmal AF (PAF) and 142 (33.2\%) had persistent AF (PeAF), including 33 patients with long standing PeAF. Follow up was available for 405 (94.2\%) cases over a mean period of $20 \pm$ 10 months, with a total of 1564 follow up clinic episodes (median 4/patient) reviewed. AF recurrence was deemed to have occurred either if the arrhythmia was documented on ECG, or if the attending clinician treated the patient as for recurrence even without definitive proof, e.g. by starting new antiarrhythmic medication.

Results Major complications occurred in 6/659(0.9\%)patients, including 1 pericardial tamponade requiring pericardiocentesis, 1 pulmonary vein stenosis, 2 right phrenic nerve palsies, and 2 cases of groin hematoma requiring blood transfusion. No stroke, TIA, femoral vascular intervention, atrio-oesophageal fistula or death occurred and no case required thoracotomy or extended hospital stay beyond 2 days. Small hematomas, not requiring blood transfusion but delaying hospital discharge by a day, occurred in $9(1.4 \%)$ patients.

Clinical freedom from AF for PAF was 55\% after single procedure and $86 \%$ after a mean 1.3 (1-3) procedures, and for PeAF was $42 \%$ after single procedure and $74 \%$ after a mean $1.4(1-3)$ procedures. $60(15 \%)$ of the patients were on anti-arrhythmic drugs at last follow up, compared to $279 / 400$ (70\%) at time of AFA.

Conclusions Complication rates for AFA have decreased markedly in modern practice. Clinical success rates of $85 \%$ in PAF and $75 \%$ in PeAF can be expected with Contact Force sensing RF catheters, although to achieve these, $30-40 \%$ of patients require more than one procedure, and $15 \%$ patients need adjuvant AAD. 